
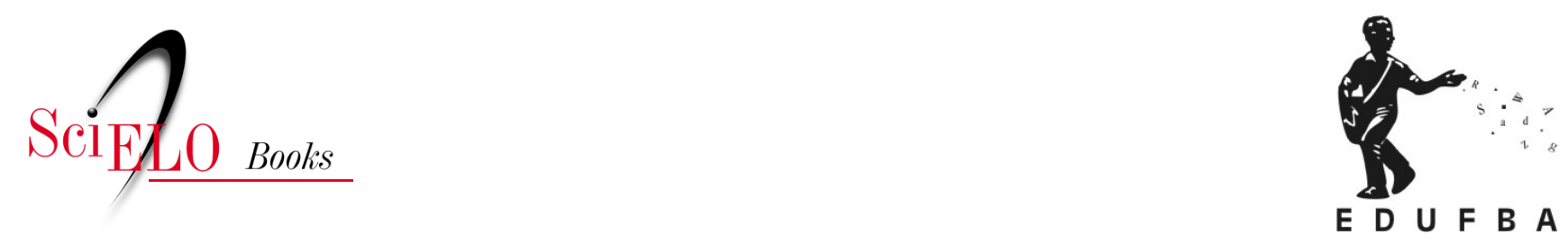

\title{
1 - Respostas governamentais à epidemia da aids situando a problemática e o objeto da pesquisa
}

\author{
Sandra Garrido de Barros
}

\section{SciELO Books / SciELO Livros / SciELO Libros}

BARROS, S.G. Respostas governamentais à epidemia da aids: situando a problemática e o objeto da pesquisa. In: Política Nacional de Aids: construção da resposta governamental à epidemia HIV/aids no Brasil [online]. Salvador: EDUFBA, 2018, pp. 16-26. ISBN 978-85-232-2030-3.

https://doi.org/10.7476/9788523220303.0002.

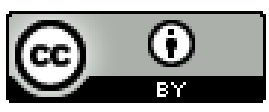

All the contents of this work, except where otherwise noted, is licensed under a Creative Commons Attribution 4.0 International license.

Todo o conteúdo deste trabalho, exceto quando houver ressalva, é publicado sob a licença Creative Commons Atribição 4.0. 



\section{Respostas governamentais à epidemia da aids: situando a problemática e o objeto da pesquisa}

A resposta à epidemia da aids ${ }^{1}$ no mundo teve início em um momento em que o saber médico ainda não tinha uma resposta terapêutica eficaz (PINELL et al., 2002)2002, de modo que se observava internacionalmente uma variedade de respostas governamentais determinadas, dentre outros fatores, pelo momento de identificação dos primeiros casos, pela percepção da doença em cada sociedade - quem é acometido, quantas pessoas, quais danos causados pela epidemia, natureza da disseminação dos casos -, pelo perfil epidemiológico, pelas condições políticas e econômicas de cada país e pelo estágio do desenvolvimento do conhecimento médico acerca da doença, como pode ser identificado nos estudos acerca da resposta governamental em países como Cuba (ARAZOZA et al., 2007; PÉREZ-STABLE, 1991, 1992), França (PINELL et al., 2002), China (WU et al., 2007; WU; ROU; CUI, 2004), Tailândia (AINSWORTH; BEYRER; SOUCAT, 2003), Paquistão (RAI et al., 2007), Bangladesh (AZIM et al., 2008), Suíça (VOEGTLI, 2009), Camarões (BOYER et al., 2011) e Brasil. (GALVÃO, 2000; GUERRA, 1993; MARQUES, 2003)

Poucos países anteciparam a resposta governamental aos primeiros casos de aids, como Cuba, que em 1983 instituiu as primeiras medidas para o controle da epidemia e teve o primeiro caso identificado em dezembro

No desenvolvimento do texto, optou-se por utilizar a palavra aids grafada em letras minúsculas. Apesar de ter origem na sigla AIDS, da expressão em inglês Acquired Immune Deficiency Syndrome, na língua portuguesa, siglas com quatro ou mais letras devem ser escritas apenas com a inicial maiúscula, quando formam uma palavra pronunciável. Mas, pelo seu uso, o termo aids terminou adquirindo a condição de substantivo, como nome de doença, devendo ser grafado apenas com letras minúsculas. 
de 1985 entre homossexuais; e Bangladesh, que desde 1985já havia instituído um Comitê Nacional de Aids, mas que só teve registro de casos a partir de 1989. (ARAZOZA et al., 2007; AZIM et al., 2008) Na maioria dos países, o desenvolvimento de uma política visando o controle da epidemia da aids aconteceu após a identificação dos primeiros casos no país, sendo que em alguns deles, como na França (PINELL et al., 2002), na Suíça (VOEGTLI, 2009) e no Brasil (GALVÃO, 2000; GUERRA, 1993; MARQUES, 2003), por exemplo, com importante participação de movimentos sociais e a constituição de associações específicas de luta contra a aids desde a década de 1980, enquanto em outros países essa articulação entre governo e organizações não governamentais ocorreu principalmente na década de 1990, com indução governamental, como em Bangladesh (AZIM et al., 2008) e na Tailândia. (AINSWORTH; BEYRER; SOUCAT, 2003)

Alguns países, como Cuba, Tailândia e Suíça, responderam inicialmente através de ações de controle, a exemplo da testagem massiva para identificação de casos e isolamento de soropositivos. (AINSWORTH; BEYRER; SOUCAT, 2003; ARAZOZA et al., 2007; PÉREZ-STABLE, 1991, 1992; VOEGTLI, 2009)

No Brasil, na resposta inicial à epidemia, prevaleceram estratégias com ênfase na promoção da saúde e prevenção, como educação em saúde e estímulo ao uso de preservativos. (GALVÃO, 2000; GUERRA, 1993; PIOT; SECK, 2001) A partir de 1989 essas estratégias foram associadas ao fornecimento de zidovudina (AZT) no estado de São Paulo, e em 1991 pelo Ministério da Saúde. (PROGRAMA NACIONAL DE DST E AIDS, 2005) A associação de ações de promoção da saúde e prevenção da doença à oferta de medicamentos também foi o caminho adotado em países onde a doença se manifestou mais tardiamente, como na China, Paquistão e Camarões, por exemplo. (BOYER et al., 2011; RAI et al., 2007; WU et al., 2007)

As notícias acerca da nova doença chegaram antes da identificação dos primeiros casos de aids no país, através da imprensa, que além da influência na sua divulgação, no alerta ao seu surgimento e no despertar do interesse de profissionais de saúde e grupos sociais, que começaram a se mobilizar, também disseminou metáforas que contribuíram para criar um comportamento de preconceito, medo, moralismo, pânico e 
até mesmo de indiferença, como se esse fosse um problema estrangeiro. (MARQUES, 2003) A nova doença era denominada por nomes como “doença dos homossexuais”, "câncer gay”, "praga gay”, "peste gay”. (GALVÃO, 2000; MARQUES, 2003)

Os primeiros casos foram identificados na cidade de São Paulo, a partir de 1982,² e em meados de 1983, a Secretaria de Saúde do Estado já estava implantando o primeiro programa governamental de luta contra a aids no país. (FRANÇA, 2008; GALVÃO, 2000; GUERRA, 1993; MARQUES, 2003) No âmbito nacional, apenas a partir de 1985, a aids entrou na agenda política do Ministério da Saúde. (BRASIL, 1985b; GALVÃO, 2000)

Os princípios de universalidade do acesso, integralidade das ações e participação social propostos pelo movimento da Reforma Sanitária Brasileira e inseridos na Constituição Federal de 1988 orientaram a formulação e implantação da resposta brasileira à epidemia. (GRANGEIRO; SILVA; TEIXEIRA, 2009; MALTA; BASTOS, 2008) Desde 1991, o programa nacional passou a fornecer AZT para pessoas vivendo com HIV/aids. A partir de 1996, a distribuição universal de antirretrovirais (ARV), estratégia do programa nacional reconhecida internacionalmente, foi incorporada à política brasileira. Entre outros fatores, em decorrência da pressão social exercida pelos inúmeros processos judiciais propostos por pessoas vivendo com HIV/aids, contra as três esferas do governo, para ter acesso à terapia ARV através do Sistema Único de Saúde. (GALVÃO, 2000; MALTA; BASTOS, 2008; PROGRAMA NACIONAL DE DST E AIDS, 2005)

Entre 1980 e junho de 2015, foram registrados no país 798.366 casos de aids. ${ }^{3}$ (BOLETIM..., 2015) Nos primeiros anos, a epidemia atingiu os homossexuais masculinos dos grandes centros urbanos da região Sudeste, principalmente São Paulo, posteriormente difundindo-se para todos os estados e para a população em geral, e nos estratos sociais de

2 Um caso foi registrado retrospectivamente como ocorrido em 1980. (TEIXEIRA, 1997)

3 Casos notificados no Sistema de Informações sobre Agravos de Notificação Compulsória (Sinan) e registrados no Sistema de Controle de Exames Laboratoriais (Siscel) e no Sistema de Controle Logístico de medicamentos (Sisclom) até 30/06/2015 e declarados no Sistema de Informações de Mortalidade (SIM) de 2000 a 2010, dados preliminares para os últimos cinco anos. 
maior nível de escolaridade. (BASTOS; BARCELLOS, 1995; FONSECA; SZWARCWALD; BASTOS, 2002; SZWARCWALD et al., 2000)

A estimativa de prevalência de HIV/aids, em indivíduos de 15 a 49 anos, tem se mantido estável em 0,6\% desde 2004- - ,4\% entre as mulheres e $0,7 \%$ entre os homens (BOLETIM..., 2014), ficando bem abaixo da expectativa do Banco Mundial para o ano 2000 no momento da implantação do primeiro acordo de empréstimo, que previa 1.200.000 brasileiros infectados. (THE WORLD BANK, 1993) A taxa de incidência encontra-se estabilizada no país desde 2000, tendo sido de 17,9 por 100.000 habitantes em 2010. (BOLETIM..., 2011; SZWARCWALD et al., 2000)

A tendência à estabilização da epidemia teve início em 1997, coincidindo com a introdução da terapia antirretroviral universal no país. (DOURADO et al., 2006) Desde 1996, a distribuição gratuita e universal de medicamentos constituiu-se em componente central do programa nacional, a despeito do fato de que o Banco Mundial, principal agência financiadora das políticas de HIV/aids no mundo, priorizava a prevenção em detrimento de gastos com assistência e tratamento considerados caros, em especial nos países em desenvolvimento. (GALVÃO, 2000; MALTA; BASTOS, 2008; OISG, 2008; THE WORLD BANK, 1993)

Entre 1980 e 2010 , ocorreram alterações na participação das categorias de exposição à doença. Se no início da epidemia prevalecia a categoria de homens que fazem sexo com homens (homo e bissexuais), seguida pelos hemofílicos e receptores de sangue ou hemoderivados, a partir de 1989 observou-se um aumento da participação das subcategorias usuários de drogas injetáveis (UDI) e transmissão heterossexual. (BOLETIM..., 2001)

Entre 1991 e 1993, os UDI passaram a ser a categoria predominante, sendo que, a partir de 1994, a principal categoria de exposição passou a ser a heterossexual (BOLETIM..., 2001), levando também a um maior acometimento do sexo feminino. (BASTOS; BARCELLOS, 1995) Assim, a razão de sexo (M:F) que era de 40:1 em 1983, a partir de 2002, estabilizou-se em 1,5:1. (BOLETIM..., 2009) Desde 2000, a epidemia passou a ser classificada como estabilizada e concentrada em populações de maior vulnerabilidade. (BOLETIM..., 2011) 
A preocupação com a oferta de medicamentos, desde o final da primeira década de implantação, aponta para uma especificidade da política brasileira: a incorporação da diretriz da integralidade da atenção, que busca articular prevenção e tratamento. Característica esta que, associada ao enfrentamento dos preços impostos pela indústria farmacêutica, contribuiu também para a implantação dos medicamentos genéricos no Brasil. (LOYOLA, 2008; RODRIGUES; SOLER, 2009)

Nesse sentido, o Brasil assumiu importante papel na luta pela redução dos preços dos medicamentos ARV, evidenciada pela disputa entre 2000 e 2001 no âmbito da Organização Mundial do Comércio (OMC) com os Estados Unidos da América pela questão da licença compulsória de medicamentos e mais recentemente, em 2007, pela efetivação da licença compulsória do efavirenz. (LOYOLA, 2008; RODRIGUES; SOLER, 2009)

O Programa Nacional de DST/Aids tem sido considerado como de bom desempenho e impacto sobre indicadores de morbimortalidade, ainda que apresente limitações no que tange à manutenção e controle do estoque de medicamentos, infraestrutura dos serviços, treinamento adequado para profissionais de saúde, além das questões relacionadas ao financiamento. (MALTA; BASTOS, 2008; PIOT; SECK, 2001) Principalmente em função dos resultados obtidos com a distribuição universal de ARV (HACKER et al., 2004; OLIVEIRA-CRUZ; KOWALSKI; MCPAKE, 2004), a política brasileira de controle da epidemia de aids tem sido citada como modelo não apenas para outros países em desenvolvimento como Costa Rica, El Salvador, Panamá (PIOT; SECK, 2001) e China (WU et al., 2007), mas também para os próprios Estados Unidos da América (EUA). (GÓMEZ, 2008, 2010; NUNN, 2009) Podem ser citados como exemplos de iniciativas que sofreram influência da estratégia brasileira:

- o President's Emergency Plan for AIDS Relief (PEPFAR), ${ }^{4}$ através do qual os EUA fornecem medicamentos para aids para países africanos e caribenhos;

4 Iniciativa do governo norte-americano para reduzir o sofrimento das pessoas vivendo com HIV/aids no mundo que teve início em 2003, cujo um dos objetivos é expandir prevenção, cuidado e tratamento. (PEPFAR, 2012) 
- a iniciativa 3 by $5^{5}$ da Organização Mundial de Saúde (OMS), que previa um incremento de 3 milhões de pessoas fazendo uso de ARV no final de 2005. (OKIE, 2006)

O interesse internacional pela política brasileira de controle da aids pode ser evidenciado através de artigos e estudos publicados na literatura internacional por pesquisadores estrangeiros, descrevendo o desenvolvimento da estratégia nacional, inclusive em comparação com a política norte-americana e enfatizando não apenas a estratégia de acesso universal aos medicamentos ARV, mas também as estratégias de prevenção adotadas pelo Programa Nacional. (GÓMEZ, 2008, 2010; NUNN, 2009; OKIE, 2006)

Estudos que tratam da resposta à epidemia e da gênese da política têm dado ênfase à análise da participação das organizações não governamentais (ONGs) na luta contra a aids no país ou da sua relação com o Estado, sempre colocando o campo burocrático em um segundo plano. (CAMPOS, 2005; GALVÃO, 2000; MONTEIRO, 2006; SILVA, 1999) Aqueles que fizeram uma análise mais centrada na resposta governamental à epidemia também não estenderam a sua análise à participação do campo médico e científico. (GÓMEZ, 2008; MARQUES, 2003; NUNN, 2009) Além disso, outras investigações têm valorizado principalmente o processo de implementação da política estatal, tratando de períodos, contextos ou aspectos mais específicos, analisando os processos de formulação e desenvolvimento da política no estado de São Paulo, para usuários de drogas injetáveis, acerca da descentralização da política, ou realizando estudos do grau de implantação da política em unidades federativas e acerca da institucionalização do programa em municípios. (BARBOZA, 2006; FONSECA, 2008; FRANÇA, 2008; GUERRA, 1993; LIMA, 2006; SANTOS, 2009; STANISE, 2008)

Na França, Pinell e colaboradores (2002) operacionalizando o conceito de espaço social, estudaram o universo de possíveis para a emergência de um movimento de luta contra a aids naquele país, assim como a estrutura e a dinâmica de relações dos agentes engajados no espaço aids medicamentos ARV a 3 milhões de pessoas vivendo com HIV/Aids até 2005. (WHO, 2012) 
no período de 1981 a 1996. O espaço associativo aids na França surgiu com a mobilização popular frente a lacunas e incoerências das medidas governamentais para o enfrentamento da epidemia, ou seja, buscando substituir as instituições estatais para cumprir uma função pública. A luta contra a aids, ao contrário do combate a outras doenças como câncer e a tuberculose, também estudadas por Pinell (2010), assumiu, assim, características de contestação social, estruturada por associações militantes. A criação de associações específicas de luta contra a aids foi anterior à resposta governamental e teve como principais agentes militantes homossexuais, sendo que aquele espaço associativo conformou-se com uma autonomia relativa em relação ao campo homossexual, o que permitiu um alargamento da base do movimento e a abertura de serviços aos demais grupos atingidos pela epidemia. A partir da atuação dessas associações, dos avanços no campo médico e da evolução da representação da doença - que passou a atingir a população geral - pela sociedade francesa, foi formulada a política de luta contra a aids daquele país, provocando modificações importantes em áreas como a homossexualidade e a toxicomania, considerando a sua associação com a epidemia.

No Brasil foi localizado apenas um trabalho que buscou articular a posição ocupada no "campo de políticas públicas em HIV/aids” ou no “campo do HIV/aids" e as formas de ação empreendedora, ${ }^{6}$ baseado no conceito de campo proposto por Bourdieu. (MENDONÇA; ALVES; CAMPOS, 2010) O estudo considerou o grupo de médicos que assumiu posições importantes no programa nacional, em especial os de São Paulo, e as organizações não governamentais como empreendedoras institucionais, sendo as últimas empreendedoras desafiantes por ocuparem uma posição periférica, além de terem importante papel na manutenção da legitimidade do tema fora do "campo do HIV/aids" (empreendedorismo extrainstitucional). A análise de Mendonça, Alves e Campos (2010) concentrou-se especialmente no campo médico e nas associações específicas de luta contra a aids, considerando outsiders a população geral e as demais áreas

6 Otrabalho desenvolvido por Mendonça, Alves e Campos (2010) teve como principal referencial a teoria organizacional neo-institucionalista. 
do setor saúde; o campo científico e o campo burocrático. Além disso, o estudo também não explorou a potencialidade de análise do referencial bourdieusiano, principalmente na interação entre trajetória dos agentes e as condições históricas de possibilidade.

Nesse sentido, entende-se que o conceito de espaço aids, sugerido por Pinell e colaboradores (2002), mostra-se mais adequado que o de campo, haja vista tratar-se de um espaço de relações entre agentes envolvidos com a organização da resposta à epidemia da aids, ainda que tenha contribuído para a constituição de um movimento associativo específico ao seu interior, dependente do conhecimento médico acerca da doença - definição das causas, descoberta de testes diagnósticos, possibilidades terapêuticas - para seu desenvolvimento. Ou seja, o espaço aids tem uma relação de dependência, em especial com o campo médico, não podendo ser compreendido como um campo que possui autonomia relativa, conforme proposto por Bourdieu.

Os estudos revisados concentraram-se na análise de casos de unidades federativas, em especial São Paulo, ou em uma abordagem nacional centrada na relação entre ONGs e Estado, seja destacando a atuação política dessas organizações, seja enfatizando a formulação e implantação da política pública nos diferentes níveis do sistema de saúde. Os referidos trabalhos não têm analisado de forma mais sistemática a interação entre os agentes dos diferentes subespaços envolvidos na resposta à epidemia da aids no país, seus pontos de vista sobre a epidemia e as estratégias adotadas na emergência do espaço aids e na formulação e consolidação da política específica de luta contra a doença no país. Dessa forma, diversas perguntas não estão respondidas nas investigações revisadas:

- Por que foi formulada uma política para o controle da aids logo após a sua descoberta, quando não havia evidências sobre a sua magnitude e vulnerabilidade?

- Como e quando se desenvolveu no Brasil uma política de controle da aids baseada não apenas na prevenção, mas também no tratamento (doenças oportunistas e, mais tarde, acesso a ARV)? 
- Como se organizou o espaço aids no Brasil? Qual a sua composição? Quem são seus agentes? O que estava em disputa? Por que as pessoas interessaram-se pela luta contra a aids? Qual o impacto sobre o movimento homossexual? Quais os interesses de cada subespaço específico? Quais as relações de poder estabelecidas?

- Qual a relação das ações de luta contra a aids e o movimento pela Reforma Sanitária brasileira?

À medida que a história avança, o espaço de possíveis realizáveis se fecha, porque as alternativas iniciais de onde saíram as escolhas historicamente construídas são esquecidas, o que Bourdieu (2012, tradução nossa) denominou "amnésia da gênese". As escolhas e decisões tornamse constrangimentos objetivados nas instituições, e interiorizados e incorporados pelos agentes. De modo que, para este autor, a análise sócio -histórica, além de identificar os agentes e disputas iniciais, pode revelar os possíveis não realizados, mortos e esquecidos, afastando-nos da tendência em aceitar que o ocorrido era o que deveria ter acontecido. O estudo da gênese, então, é uma importante estratégia de ruptura com o senso comum e a análise, a partir do referencial teórico bourdieusiano, permite a articulação entre estruturas objetivas e mentais, internas e externas, e as trajetórias dos agentes envolvidos, buscando uma integração entre o material e o simbólico.

O estudo da gênese e consolidação da política nacional de controle da aids no Brasil, como caso exemplar do possível, apoiado nesse referencial teórico, pode fornecer elementos para a resposta a algumas dessas indagações, a partir da análise das condições históricas que contribuíram para a constituição do espaço aids no país e da trajetória social e profissional de participantes desse processo. Nesse sentido, foram realizadas 31 entrevistas em profundidade com agentes envolvidos na luta pela resposta à epidemia da aids no Brasil, ocupando diferentes posições no interior do espaço aids, em diversos momentos históricos, além de uma consulta por e-mail, bem como análise de documentos do Ministério da Saúde, de organizações não governamentais e reportagens veiculadas na mídia impressa. ${ }^{7}$

7 O desenho do estudo encontra-se detalhado nos Apêndices A a C. 
O resultado desse estudo mostra que, no Brasil, o fato de a gênese da política de controle da aids ter ocorrido durante um momento de ascensão do movimento sanitário e na ausência de uma terapia específica, pode ter resultado na priorização inicial da prevenção, mas sempre sob a dominância do campo médico, levando, a partir da década de 1990, à ênfase na universalização do tratamento. $O$ fato de ter inicialmente atingido um grupo organizado, como os homossexuais, e com lideranças com alto volume de capital cultural e social, portanto com uma importante rede de contatos, contribuiu para uma resposta precoce por parte do Estado brasileiro, quando ainda não havia evidências epidemiológicas da magnitude e vulnerabilidade da doença no país.

A emergência dessa política durante o período de redemocratização, com a formação de várias organizações não governamentais, e a constituição do Sistema Único de Saúde, quando agentes do movimento sanitário assumiram importantes funções nos diferentes níveis da gestão do sistema de saúde brasileiro, bem como a dominância do campo médico podem ter contribuído para a formulação de uma política baseada na integralidade das ações e no acesso universal à assistência, como podem ter possibilitado uma maior participação da burocracia estatal na sua formulação.

Isso foi possível de ser verificado a partir da análise da gênese e consolidação da política nacional de controle da aids no Brasil, no período compreendido entre 1981 e 2001, bem como das trajetórias dos agentes envolvidos com a formulação e implantação dessa política, das relações estabelecidas entre esses agentes com o espaço da saúde coletiva, o movimento da reforma sanitária brasileira, o campo médico e o campo do poder. Também foram analisadas as condições de possibilidade históricas e o universo de possíveis que permitiram a formulação de uma política baseada na integralidade e na universalidade da atenção à saúde. 